\title{
The Availability of Quantitative Assessment of Pain Perception in Patients With Diabetic Polyneuropathy
}

\author{
Tae Jun Park, MD, Sung Hoon Kim, MD, PhD, Hi Chan Lee, MD, \\ Sae Hoon Chung, MD, Ji Hyun Kim, MD, Jin Park, MD
}

Department of Rehabilitation Medicine, Yonsei University Wonju College of Medicine, Wonju, Korea

\begin{abstract}
Objective To evaluate the usefulness of the quantitative assessment of pain perception (QAPP) in diabetic polyneuropathy (DPN) patients.

Methods Thirty-two subjects with DPN were enrolled in this study. The subjects' pain perception was assessed quantitatively. Current perception threshold (CPT) and pain equivalent current (PEC) were recorded. All patients were tested with a nerve conduction study (NCS) for evaluation of DPN and pain-related evoked potential (PREP) for evaluation of small fiber neuropathy (SFN) on bilateral upper and lower limbs. All patients were asked to participate in tests such as visual analogue scale (VAS) and SF-36 Health Survey Version 2 to evaluate their subjective pain and quality of life, respectively.

Results The PEC of QAPP showed significant correlations with VAS $(\mathrm{p}=0.002)$ and physical function surveyed with SF-36 Health Survey Version $2(\mathrm{p}=0.035)$. The results of QAPP had no correlation with NCS, but there was a significant relationship between the CPT of QAPP and PREP $(\mathrm{p}=0.003)$.

Conclusion The QAPP may be useful not only in providing objective evaluations of subjective pain in patients with DPN but also in the assessment of diabetic SFN.
\end{abstract}

Keywords Diabetic neuropathies, Small fiber neuropathy, Pain measurement, Nociceptive pain

\section{INTRODUCTION}

According to the Diabetes Atlas, published by the International Diabetes Federation in 2014, an estimated
$8.3 \%$ of the world's population are patients with diabetes mellitus (DM) [1]. Diabetic polyneuropathy (DPN) is a common complication of diabetes and is one of the most common causes of peripheral neuropathy [2]. DPN pro-

Received July 12, 2017; Accepted September 6, 2017

Corresponding author: Jin Park

Department of Rehabilitation Medicine, Yonsei University Wonju College of Medicine, 20 Ilsan-ro, Wonju 26426, Korea. Tel: +82-33-741-0664, Fax: +8233-742-1409, E-mail: lighthouse14@naver.com

ORCID: Tae Jun Park (http://orcid.org/0000-0003-4835-5778); Sung Hoon Kim (http://orcid.org/0000-0001-6043-7640); Hi Chan Lee (http://orcid. org/0000-0002-5116-094X); Sae Hoon Chung (http://orcid.org/0000-0002-7861-9503); Ji Hyun Kim (http://orcid.org/0000-0001-9061-3350); Jin Park (http://orcid.org/0000-0001-5751-9610).

@ This is an open-access article distributed under the terms of the Creative Commons Attribution Non-Commercial License (http://creativecommons.org/ licenses/by-nc/4.0) which permits unrestricted noncommercial use, distribution, and reproduction in any medium, provided the original work is properly cited. Copyright $\odot 2018$ by Korean Academy of Rehabilitation Medicine 
gresses in a length-dependent manner, and most of the symptoms originally manifest in both feet and progress to both hands. Motor symptoms are preceded by sensory symptoms. With regard to sensory symptoms, abnormal pain and temperature sensations precede vibration sensations. This implies that small nerve fibers are compromised before large nerve fibers [3].

Until now, nerve conduction studies (NCS) have been widely used to confirm the diagnosis of DPN along with clinical symptoms or signs of neuropathy. However, many diabetic patients show symptoms and signs of nerve damage despite normal NCS [4]. Although NCS is a very sensitive test used to measure the function of large myelinated nerve fibers, it is limited by the fact that it is unable to detect autonomic nerve damage or small fiber neuropathy (SFN), which is common in diabetic patients [5]. According to some studies, the pain-related evoked potential (PREP), which is induced by nociceptive electrical stimulation of the skin, is useful for the early diagnosis of diabetic SFN [6]. There is a need for methods of evaluation that can more easily reflect the condition of patients, thereby assisting in the diagnosis of diabetic SFN.

The main symptoms that patients with DPN complain of and wish to have managed are sensory symptoms, such as tingling sensations and pain. However, since pain is subjective, it is difficult to measure it and evaluate the degree of discomfort a patient is experiencing. The most common methods for evaluating pain in clinical practice include the visual analogue scale (VAS), numerical rating scale, McGill Pain Questionnaire, electrophysiological method (Neurometer, etc.), and thermography. However, none of these methods provide ways of evaluating the intensity of pain objectively [7-10].

Methods for objective and quantitative evaluation of diabetic neuropathy have been investigated by many researchers in order to diagnose diabetic neuropathy and observe its response to treatments. One of these methods, the quantitative sensory test (QST), was developed as a way to quantitatively measure mechanical stimuli, such as thermal stimulus, vibration, and pinprick sense. Although the QST is known to be helpful in early diagnosis of sensory neuropathies such as DPN, there remains a problem, in that the normal limits of the QST have not yet been established [11].

Recently, a method of assessing the pain in a quantitative manner using heteresthesia has been developed and applied in clinical practice to various patients complaining of pain [12]. Quantitative assessment of pain perception (QAPP) is a quantitative sensory test that uses esthesiometer, applying current to test the sensory function of patients. With it, pain perception can be quantified by comparing the magnitude of the sensation caused by painless electrical simulation with the magnitude of current that stimulates a certain quantity of the sensation corresponding to the pain.

In this study, we aimed to investigate the usefulness of QAPP in DPN.

\section{MATERIALS AND METHODS}

\section{Subjects}

The inclusion criteria were adults older than 20 years who were diagnosed with DM and experienced neuropathic sensory symptoms. After ruling out other causes of peripheral polyneuropathy, typical clinical symptoms alone can be used to diagnose DPN [13]. Accordingly, all the participants enrolled in this study can be regarded as having been diagnosed with DPN. The exclusion criteria in this study were a low level of consciousness without the capacity to fully express intentions, acute diseases, malignant tumor, chronic alcoholism, or a history of central nervous system disorders, such as head injury or cerebrovascular disease, or a history of other peripheral neuropathies, such as carpal tunnel syndrome (positive Tinel test or positive Phalen test), or radiculopathy (positive foraminal compression test or positive Spurling test). Also excluded were subjects with illness that might cause other peripheral polyneuropathies, those with diseases requiring hospitalization or surgery, those with electrical allergy, pregnant women, and other participants deemed inappropriate by the research director.

All subjects voluntarily participated in this study after receiving sufficient explanations, and all signed an informed consent form approved by the Institutional Review Board of Wonju Severance Christian Hospital (No. CR215008).

\section{Quantitative assessment of pain perception \\ Definition}

This is a test method that compares the magnitude of the sensation caused by painless electrical simulation with the magnitude of current that stimulates a certain 
quantity of the sensation corresponding to the pain in order to quantify the perception of pain.

\section{Stimulated current}

For electrical stimulation we applied pulsed current to patients. The frequency of the current was $50 \mathrm{~Hz}$, which would stimulate primarily $A \beta$ fibers and some $A \delta$ fibers among many nerve fibers. The pulse width was set to 0.3 $\mathrm{ms}$, and the power of the current was between 0-15 $\mathrm{mA}$.

\section{Measurement method}

Pain perception was evaluated using the PV-300 (PainView, Ossein Co. Ltd., Wonju, Korea). While the patient was in a comfortable sitting position, a $1.5 \mathrm{~cm} \times 5 \mathrm{~cm}$ electrode patch, which was connected to the patch port, was attached to the left anterior forearm of the subject, and a stop switch was held in the right hand of the subject (Fig. 1). After positioning the subject so that the monitor of the measuring device could not be seen by the subject, a mixed sense of electrical stimulation was applied to the site to which the electrode patch was attached. The power of the electrical stimulation started at zero, and during the examination the power was slowly increased. Subjects were instructed to press the stop switch when they started to feel any sensation on the patch attachment site. This threshold for electrical stimulation was defined as the current perception threshold (CPT). The subject was also instructed to press the stop switch when they felt a sensation similar to the usual neuropathic

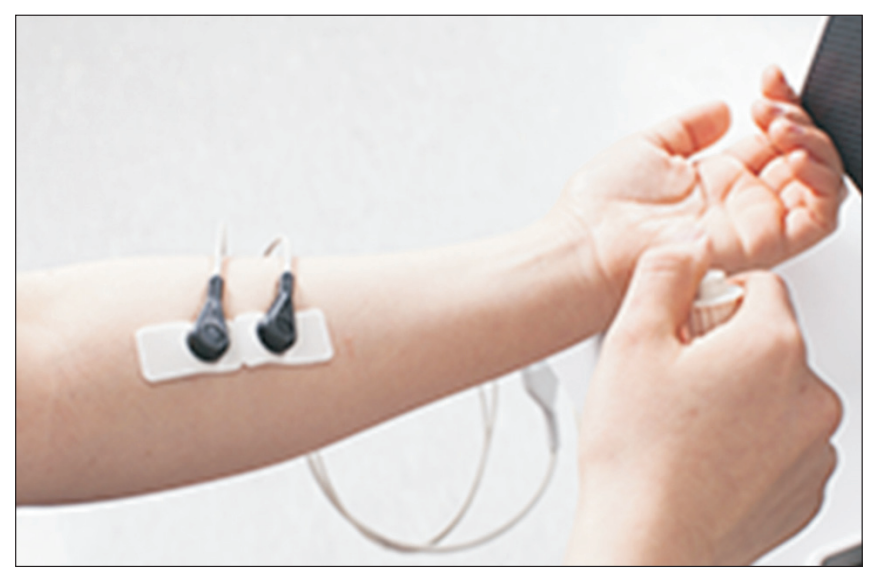

Fig. 1. Measuring position for quantitative assessment of pain perception. While in a comfortable seating position, the electrode patch is attached to the left anterior forearm and the stop switch is held in the right hand. pain in their limbs. This indicates the magnitude of the current that created a sensation corresponding to pain, otherwise called pain equivalent current (PEC). The CPT and PEC were measured three times in each subject, and the mean values were recorded.

\section{Quantification of pain}

When measuring the CPT, the pain degree was calculated with the formula of $100 \times(\mathrm{PEC}-\mathrm{CPT}) / \mathrm{CPT}$, in order to eliminate the difference induced by relative location between electrodes and subcutaneous nervous system, or individual variation in perceiving electrical stimulation in the brain. The CPT and PEC were measured three times in each subject, and the mean values were substituted for the calculation.

\section{Nerve conduction study}

NCS was performed on the upper and lower limbs of both sides while the subjects were in a comfortable lying position. NCS was tested using the Dantec Keypoint Portable Electromyography System (Alpine Biomed, Skovlunde, Denmark). The compound muscle action potential (CMAP) and sensory nerve action potential (SNAP) of the median and ulnar nerves were measured in the upper extremities, and the CMAP and SNAP were measured in the lower extremities for the tibial and peroneal nerves and for the sural and superficial peroneal nerves, respectively. Onset latency, peak-to-peak amplitude, and conduction velocity were recorded for each measurement. In addition, the number of abnormal NCS results for each subject and the number of nerves observed to be abnormal in any of the NCS measurements (onset latency, peak-to-peak amplitude, and conduction velocity) were counted.

\section{Pain-related evoked potential}

PREP was performed on all subjects in a comfortable lying position on bilateral upper and lower limbs, using the Dantec Keypoint Portable Electromyography System (Alpine Biomed). Custom-built concentric surface electrode samples manufactured by a medical engineering company (Hurev Co. Ltd., Wonju, Korea) were used as the stimulating electrodes. The electrodes featured a central cathode ( $0.5 \mathrm{~mm}$ in diameter) and an external anode ring (6 $\mathrm{mm}$ in diameter). The custom-built electrode used a conventional carbon wire cable and a socket, which fit- 
ted well with the portable electromyography system. For each subject, the stimulation was raised and lowered slowly, and the stimulation threshold was determined at a level where the subject perceived pain continuously. Then, 22 double pulses were applied at an intensity of 1.5 times the individual pain threshold (monopolar square wave: double pulse interval of $5 \mathrm{~ms}$; duration of $0.5 \mathrm{~ms}$; interstimulus interval of 16 to 17 seconds). A recording electrode placed at $\mathrm{Cz}$ referred to the linked earlobes of the international 10-20 system (bandwidth of $1 \mathrm{~Hz}$ to $1 \mathrm{kHz}$; sweep length of $500 \mathrm{~ms}$ ). In the upper limb, the stimulating electrode was placed on the dorsum side of the middle phalanx of the second finger ( $\mathrm{C} 7$ dermatome). In the lower limb, the stimulating electrode was placed on the dorsum side of the middle phalanx of the second toe (L5 dermatome). N latency, P latency, and NP amplitude were recorded for each test.

\section{Evaluation of subjective pain and quality of life}

The subjective pain of individuals was evaluated using the visual analogue scale. The 36-Item Short-Form Health Survey Version 2 (SF-36v2) was used for the evaluation of quality of life.

\section{Statistical analysis}

Correlation analysis was used to analyze data. Analysis was performed using the PASW Statistics version 18.0 (SPSS Inc., Chicago, IL, USA), and statistical significance was accepted for $\mathrm{p}$-values of less than 0.05 .

\section{RESULTS}

\section{General characteristics of subjects}

Thirty-two patients (16 men and 16 women) with DPN were enrolled in this study. Four individuals were in their 20s ( 2 men and 2 women), 5 were in their 30 s ( 3 men and 2 women), 6 were in their 40s (3 men and 3 women), 7 were in their 50s ( 3 men and 4 women), and 10 of them were over 60 ( 5 men and 5 women). Ages ranged from 21 to 65 years, with an average of $48.56 \pm 14.09$ years. Height ranged from 145.0 to $181.0 \mathrm{~cm}$, with an average height of $163.90 \pm 9.55 \mathrm{~cm}$. Weight ranged from 44.0 to $92.0 \mathrm{~kg}$, with an average weight of $69.60 \pm 11.68 \mathrm{~kg}$. As for the clinical characteristics of participants, the mean duration of DM was $11 \pm 7$ years in their $20 \mathrm{~s}, 5 \pm 3$ years in their 30 s, $6 \pm 5$ years in their 40 s, $8 \pm 7$ years in their 50 s, $12 \pm 10$ years in patients over 60 . The mean duration of DM in participants of all age groups was $9 \pm 7$ years. The analysis of the mean VAS score of participants, according to age group, was $11 \pm 3$ in their 20 s, $27 \pm 17$ in their 30 s, $36 \pm 16$ in their 40 s, $66 \pm 29$ in their 50s, $37 \pm 29$ in the group over 60 (Table 1).

\section{Clinical symptoms of subjects}

Twenty-seven subjects (84\%) had bilateral neuropathic sensory symptoms, while 5 subjects (16\%) had unilateral symptoms. Also, 12 of them (38\%) had symptoms only in their feet, 9 of them (28\%) only in their hands, and 11 subjects (34\%) had symptoms in both their feet and their hands. The number of individuals who complained of a prickling feeling, burning sensation, tingling sensation, and numbness was 8 (25\%), 4 (13\%), 17 (53\%), and 3 (9\%), respectively.

\section{Quantitative assessment of pain perception}

When the QAPP in the left anterior forearm was assessed, the mean CPT was $926.6 \pm 226.3 \mu \mathrm{A}$ and the mean PEC was $1,907.4 \pm 1,382.6 \mu \mathrm{A}$. The mean pain degree was calculated as $107.71 \pm 150.46 \mu \mathrm{A}$. As for the QAPP

Table 1. Demographic and clinical variables of the participants $(n=32)$

\begin{tabular}{llccccc}
\hline & & $\begin{array}{c}\text { Sex } \\
\text { (male:female) }\end{array}$ & $\begin{array}{c}\text { Height } \\
(\mathbf{c m})\end{array}$ & $\begin{array}{c}\text { Weight } \\
(\mathbf{k g})\end{array}$ & $\begin{array}{c}\text { DM duration } \\
(\mathbf{y r})\end{array}$ & VAS \\
\hline Age (yr) & $20-29$ & $2: 2$ & $163.00 \pm 10.37$ & $58.75 \pm 10.37$ & $11 \pm 7$ & $11 \pm 3$ \\
& $30-39$ & $3: 2$ & $169.40 \pm 7.96$ & $76.00 \pm 14.37$ & $5 \pm 3$ & $27 \pm 17$ \\
& $40-49$ & $3: 2$ & $167.50 \pm 8.92$ & $77.83 \pm 15.94$ & $6 \pm 5$ & $36 \pm 16$ \\
& $50-59$ & $3: 4$ & $157.43 \pm 10.29$ & $67.86 \pm 11.82$ & $8 \pm 7$ & $66 \pm 29$ \\
\hline Average & $\geq 60$ & $5: 5$ & $163.90 \pm 9.65$ & $66.90 \pm 9.65$ & $12 \pm 10$ & $37 \pm 29$ \\
\hline
\end{tabular}

Values are presented as number or mean \pm standard deviation. DM, diabetes mellitus; VAS, visual analogue scale (0-100). 
results according to age, the average $\mathrm{CPT}$ of subjects in their 20s was $874.20 \pm 235.15 \mu \mathrm{A}$, the average PEC was $1,184.20 \pm 380.95 \mu \mathrm{A}$, and the average pain degree was $36.58 \pm 25.86 \mu \mathrm{A}$. Among the subjects in their 30 s, the average CTP was $919.30 \pm 131.93 \mu \mathrm{A}$, the average PEC was $1,940.50 \pm 1,080.47 \mu \mathrm{A}$, and the average pain degree was $115.83 \pm 128.21 \mu \mathrm{A}$. The subjects in their $40 \mathrm{~s}$ showed an average CPT of $810.50 \pm 67.40 \mu \mathrm{A}$, an average PEC of $1,354.50 \pm 624.91 \mu \mathrm{A}$, and an average pain degree of $66.57 \pm 76.05 \mu \mathrm{A}$. The average $\mathrm{CPT}$ of participants in their 50s was $921.00 \pm 226.46 \mu \mathrm{A}$, the average PEC was $2,072.80 \pm 1,761.10 \mu \mathrm{A}$, and the average pain degree was $124.06 \pm 174.67 \mu \mathrm{A}$. The average CPT among participants in their 60 s was $1,024.90 \pm 304.09 \mu \mathrm{A}$, the average PEC was $2,396.20 \pm 1,734.68 \mu \mathrm{A}$, while the average pain degree was $145.34 \pm 204.21 \mu \mathrm{A}$. Upon comparing age with CPT, PEC, and pain degree of QAPP, no statistically significant relationship was found.

There was a significant correlation between the PEC and the pain degree (correlation coefficient $=0.956$, $\mathrm{p}<0.001$ ) (Table 2, Fig. 2A). However, no statistically significant correlation was found between the duration of diabetes and CPT ( $\mathrm{p}=0.609)$, PEC $(\mathrm{p}=0.253)$, or pain degree $(\mathrm{p}=0.426)$.

\section{Correlation between VAS and OAPP}

The PEC of QAPP showed a significant correlation with the VAS (correlation coefficient $=0.526, p=0.002$ ) (Table 2, Fig. 2B). However, there was no statistically significant correlation between the CPT and the pain degree of QAPP.

\section{Correlation between Health Status and QAPP}

There was a significant correlation found between the PEC of QAPP and the physical function surveyed with the SF-36v2 (correlation coefficient $=-0.373, \mathrm{p}=0.035$ ) (Table 2, Fig. 2C).

\section{Correlation between PREP and QAPP}

The CPT of QAPP and PREP showed a significant relationship. In particular, the P latency of PREP, which was performed in the $\mathrm{C} 7$ dermatome on the left side, showed the highest correlation with the CPT (correlation coefficient $=-0.511, p=0.003$ ) (Table 2, Fig. 2D).

\section{Correlation between NCS and QAPP}

There was no significant correlation between the measurement of QAPP and the onset latency, peak-to-peak amplitude, and conduction velocity of median, ulnar, tibial, and peroneal nerves in NCS. Moreover, no significant correlation was found between the number of nerves showing abnormal findings and the results of QAPP (Table 2).

\section{DISCUSSION}

The purpose of this study was to investigate the availability of QAPP in diabetic patients with sensory symptoms. As for the main findings of this study, the results of QAPP in diabetic patients showed that there was a correlation between greater VAS and greater PEC, and between lower physical function surveyed with the SF-36v2 and greater PEC. There was no significant correlation

Table 2. Results of correlation analysis between QAPP and other evaluation tools

\begin{tabular}{lccccc} 
& \multicolumn{3}{c}{ QAPP } \\
\cline { 2 - 3 } & \multicolumn{2}{c}{ CPT } & \multicolumn{2}{c}{ PEC } \\
\cline { 2 - 3 } \cline { 5 - 6 } & Correlation coefficient & p-value & & Correlation coefficient & p-value \\
\hline Pain degree & -0.052 & 0.776 & & 0.956 & $<0.001^{*}$ \\
VAS & 0.142 & 0.395 & & 0.526 & $0.002^{*}$ \\
Physical function & a) & -0.275 & 0.128 & -0.373 & $0.035^{*}$ \\
PREP & -0.511 & $0.003^{*}$ & 0.055 & 0.766 \\
NANCS & 0.049 & 0.790 & 0.208 & 0.253 \\
\hline
\end{tabular}

QAPP, quantitative assessment of pain perception; CPT, current perception threshold; PEC, pain equivalent current; VAS, visual analogue scale (0-100); PREP, pain-related evoked potential; NANCS, number of abnormal nerve conduction study.

${ }^{a)}$ Physical function was evaluated using SF-36v2.

${ }^{*} \mathrm{p}<0.05$ by correlation analysis. 
(A)

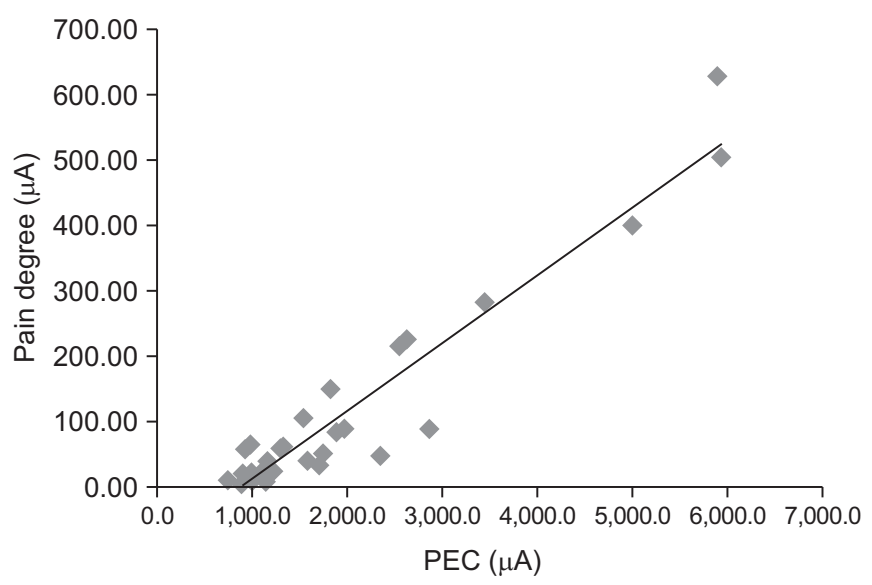

(C)

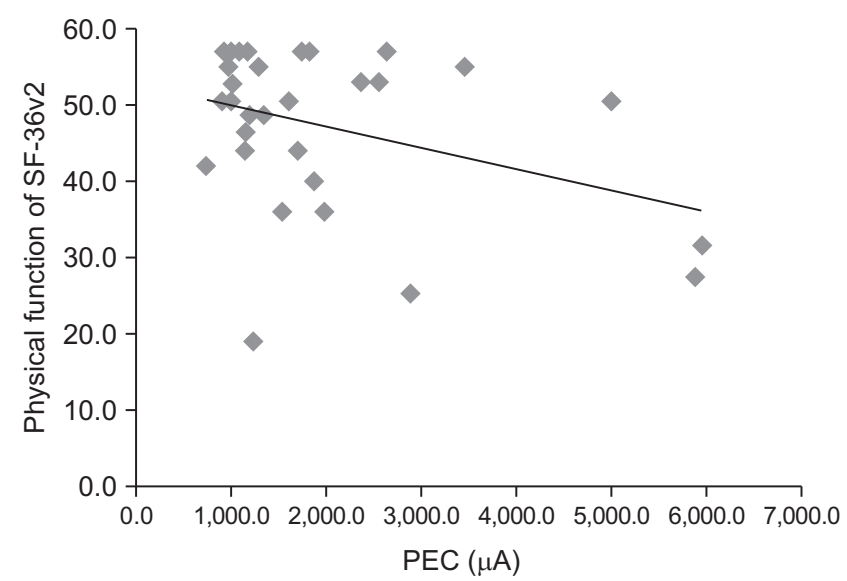

(B)

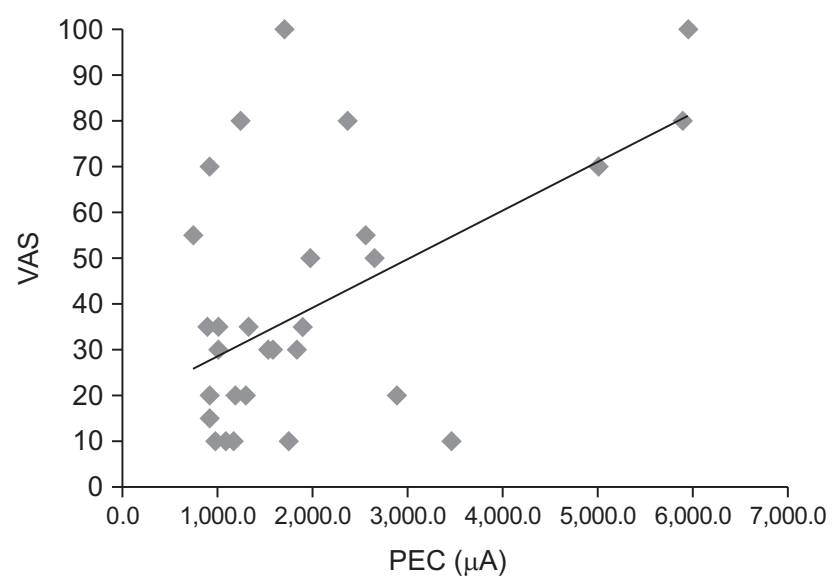

(D)

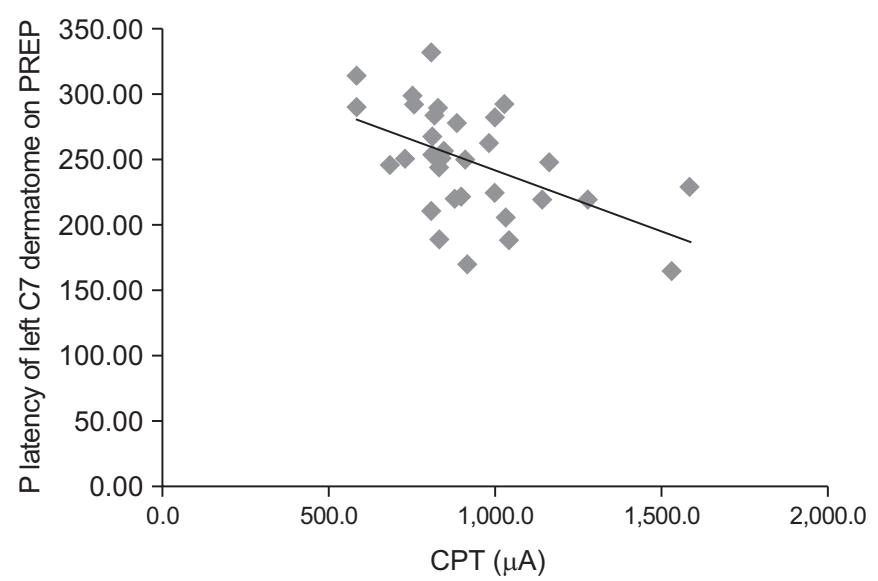

Fig. 2. (A) PEC of QAPP and pain degree showed significant positive correlation (correlation coefficient $=0.956$, $\mathrm{p}<0.0001$ ). (B) PEC of QAPP and VAS showed significant positive correlation (correlation coefficient $=0.526, p=0.002$ ). (C) PEC of QAPP and physical function in SF-36v2 showed significant negative correlation (correlation coefficient=-0.373, $\mathrm{p}=0.035$ ). (D) CPT of QAPP and P latency of PREP which was performed in C7 dermatome on the left side showed significant negative correlation (correlation coefficient $=-0.511, p=0.003$ ). PEC, pain equivalent current; QAPP, quantitative assessment of pain perception; VAS, visual analogue scale (0-100); CPT, current perception threshold; PREP, pain-related evoked potential.

between the results of QAPP and NCS. However, it was found that when the CPT was low, the P latency of PREP in the $\mathrm{C} 7$ dermatome was delayed.

Several studies have identified the VAS as a highly reliable tool for assessing pain intensity [14-16]. The SF-36v2 is known to be one of the best surveys to reflect quality of life [17]. Therefore, QAPP may well reflect the degree of subjective pain in diabetic patients.

According to the 3rd edition of the diabetic neuropathy management guidebook, published by the Korean Diabetes Association (KDA) in 2010, a patient's symptoms provide sufficient reason for a diagnosis of DPN, so that no electrophysiological studies are required, unless the cases are atypical [13]. In addition, the definitions of minimal criteria for typical DPN are organized in the update of diabetic neuropathies written by Tesfaye et al. [18] in 2010. This study defines DPN based on four criteria: (1) possible DSPN diagnosed with symptoms or signs alone; (2) probable DSPN diagnosed with symptoms and signs; (3) confirmed DSPN identified by symptoms or signs, along with an abnormal nerve conduction study or a validated measure of SFN; and (4) subclinical DSPN that shows no symptoms or signs but is diagnosed with an abnormal nerve conduction study or validated mea- 
sure of SFN. This study also recommends using criteria (1), (2), or (3) in the clinical field, and criteria (3) or (4) in research. However, since clinical symptoms or signs are subjective and qualitative in nature, NCS is widely used alternatively as an objective and quantitative test method. NCS is helpful mainly in determining the function of large myelinated nerve fibers [19]. However, DPN initially exhibits symmetrical symptoms in the lower limbs and progress to the upper extremities, affecting the small nerve fibers first, and then the large nerve fibers [20-22]. Therefore, the role of NCS in DPN is limited in the early stage [23]. With this in mind, applying a simpler and more quantitative method than NCS to evaluate small nerve fibers in DPN patients would aid in making more accurate DPN evaluations and follow-up observations after treatment.

SFN commonly involves tingling, burning, and prickling sensations, as well as shooting pain or aching due to problems with small A $\delta$ - and C-fiber functions [24]. Common causes of SFN include DM, drugs and toxic materials, infections, autoimmune diseases, inherited sensory and autonomic neuropathies, and idiopathic etiologies [25]. Methods for evaluating SFN include QST, quantitative sudomotor axon reflex testing (QSART), and skin biopsy [26]. Although QST is the most widely known and easy to perform of these methods, it alone cannot help a clinician differentiate between a problem of the central nervous system and a problem in the peripheral nervous system, and the results are not reliable when the patient has cognitive impairment. Likewise, the evaluation with QST is limited due to the fact that the patient can draw false test results [26]. Other evaluation methods for SFN include PEEP. This can be a useful tool in evaluating SFN, since it can selectively stimulate nociceptive $\mathrm{A} \delta$ - and Cfibers while avoiding the simultaneous stimulation of non-nociceptive $A \beta$-fibers among small nerve fibers [27]. QAPP is a system that evaluates $A \beta$ - and $A \delta$-fibers using stimulation frequencies [12]. In this study, the PREP was performed on the C7 dermatome, and the QAPP was performed on the C7-T1 dermatome site of the left anterior forearm. The P latency of PREP was performed on the C7 dermatome on the left side, and it was found to have the highest correlation with the CPT. Therefore, QAPP may be helpful in assisting in the diagnosis of SFN, which is a common type of DPN.

This study has several limitations. First, we examined only a small number of patients. Evaluation of a larger number of patients is needed in order to strengthen the findings of the current study. Second, this study included QAPP analysis in the absence of an evaluation for the diagnostic range of PREP in DPN patients. We could not confirm the cut off values for the diagnosis of DPN. Third, due to the exclusion criteria of the study, subjects with peripheral neuropathies, such as carpal tunnel syndrome and radiculopathy, were not assessed. In addition, those who showed positive findings in physical examinations necessary to evaluate the presence of the above neuropathies were excluded. Nevertheless, history taking and physical examination by themselves are insufficient to completely preclude those with the diseases. Finally, specific tests for the subjective evaluation of neuropathic pain were not performed, even though the study aimed to objectively evaluate neuropathic pain.

In conclusion, QAPP may be helpful in evaluating diabetic SFN as well as in objective assessment of subjective sensory symptoms in DPN patients. In addition, QAPP may be useful for the objective assessment of the progression of DPN.

\section{CONFLICT OF INTEREST}

No potential conflict of interest relevant to this article was reported.

\section{REFERENCES}

1. Aguiree F, Brown A, Cho NH, Dahlquist G, Dodd S, Dunning T, et al. IDF diabetes atlas. 6th ed. Basel: International Diabetes Federation; 2013.

2. Redmond JM, McKenna MJ, Feingold M, Ahmad BK. Sensory testing versus nerve conduction velocity in diabetic polyneuropathy. Muscle Nerve 1992;15:13349.

3. Joo IS. Controversies on the usefulness of nerve conduction study in the early diagnosis of diabetic polyneuropathy. Ann Clin Neurophysiol 2008;10:25-8.

4. American Diabetes Association American Academy of Neurology. Consensus statement: report and recommendations of the San Antonio conference on diabetic neuropathy. Diabetes Care 1988;11:592-7.

5. Park JM, Kang SJ, Kim KW, Kim JW, Kim SH. Evaluation of peripheral polyneuropathy in patients with 
diabetes mellitus using quantitative sensory test. J Korean Acad Rehabil Med 2001;25:102-9.

6. Mueller D, Obermann M, Koeppen S, Kavuk I, Yoon MS, Sack F, et al. Electrically evoked nociceptive potentials for early detection of diabetic small-fiber neuropathy. Eur J Neurol 2010;17:834-41.

7. Bradham DD. Outcomes research in orthopedics: history, perspectives, concepts, and future. Arthroscopy 1994;10:493-501.

8. Herr KA, Mobily PR, Kohout FJ, Wagenaar D. Evaluation of the Faces Pain Scale for use with the elderly. Clin J Pain 1998;14:29-38.

9. Hicks CL, von Baeyer CL, Spafford PA, van Korlaar I, Goodenough B. The Faces Pain Scale-Revised: toward a common metric in pediatric pain measurement. Pain 2001;93:173-83.

10. Johnson LL, Pittsley A, Becker R, Young AD. A novel quantitative pain assessment instrument that provides means of comparing patient's pain magnitude with a measurement of their pain tolerance. J Clin Med Res 2015;7:781-90.

11. Hansson P, Backonja M, Bouhassira D. Usefulness and limitations of quantitative sensory testing: clinical and research application in neuropathic pain states. Pain 2007;129:256-9.

12. Ohtori S, Kawaguchi H, Takebayashi T, Orita S, Inoue G, Yamauchi K, et al. PainVision apparatus is effective for assessing low back pain. Asian Spine J 2014;8:7938.

13. Chun SW, Ko KS. Summary of the update to the diabetic neuropathy management guidebook. J Korean Diabetes 2012;13:115-23.

14. Jin WJ, Yu TY, Jin YH, Lee JB. Visual analogue scale in acute pain measurement: its usefulness as a pain measurement tool in an emergency setting. J Korean Soc Emerg Med 2003;14:61-5.

15. Bijur PE, Silver W, Gallagher EJ. Reliability of the visual analog scale for measurement of acute pain. Acad Emerg Med 2001;8:1153-7.

16. Jensen MP, McFarland CA. Increasing the reliability and validity of pain intensity measurement in chronic pain patients. Pain 1993;55:195-203.

17. Jenkinson C, Wright L, Coulter A. Criterion validity and reliability of the SF-36 in a population sample. Qual Life Res 1994;3:7-12.

18. Tesfaye S, Boulton AJ, Dyck PJ, Freeman R, Horowitz M, Kempler P, et al. Diabetic neuropathies: update on definitions, diagnostic criteria, estimation of severity, and treatments. Diabetes Care 2010;33:2285-93.

19. Lee SM, Kim BJ. Diagnostic usefulness of quantitative sensory test in diabetic polyneuropathy: comparison with nerve conduction study. J Korean Neurol Assoc 1999;17:106-11.

20. Donaghue VM, Giurini JM, Rosenblum BI, Weissman $\mathrm{PN}$, Veves A. Variability in function measurements of three sensory foot nerves in neuropathic diabetic patients. Diabetes Res Clin Pract 1995;29:37-42.

21. Borsey DQ, Cull RE, Fraser DM, Ewing DJ, Campbell IW, Clarke BF. Small muscle wasting of the hands in diabetes mellitus. Diabetes Care 1983;6:10-7.

22. Ziegler D, Mayer P, Wiefels K, Gries FA. Assessment of small and large fiber function in long-term type 1 (insulin-dependent) diabetic patients with and without painful neuropathy. Pain 1988;34:1-10.

23. Jamal GA, Hansen S, Weir AI, Ballantyne JP. The neurophysiologic investigation of small fiber neuropathies. Muscle Nerve 1987;10:537-45.

24. Dyck PJ, Lambert EH, O'Brien PC. Pain in peripheral neuropathy related to rate and kind of fiber degeneration. Neurology 1976;26:466-71.

25. Cazzato D, Lauria G. Small fibre neuropathy. Curr Opin Neurol 2017;30:490-9.

26. Hovaguimian A, Gibbons CH. Diagnosis and treatment of pain in small-fiber neuropathy. Curr Pain Headache Rep 2011;15:193-200.

27. Katsarava Z, Ayzenberg I, Sack F, Limmroth V, Diener HC, Kaube H. A novel method of eliciting pain-related potentials by transcutaneous electrical stimulation. Headache 2006;46:1511-7. 